\title{
Transglutaminase 2: a new player in bronchopulmonary dysplasia?
}

\author{
Thilo J. Witsch¹, Gero Niess ${ }^{1,2}$, Elpidoforos Sakkas ${ }^{1,2}$, Tatyana Likhoshvay ${ }^{1,2}$, \\ Simone Becker ${ }^{1,2}$, Susanne Herold ${ }^{1}$, Konstantin Mayer ${ }^{1}$, István Vadász', \\ Jesse D. Roberts Jr ${ }^{3}$, Werner Seeger ${ }^{1,2}$ and Rory E. Morty ${ }^{1,2}$
}

\begin{abstract}
Affiliations: ${ }^{1}$ Dept of Internal Medicine, University of Giessen and Marburg Lung Center, Giessen, and ${ }^{2}$ Dept of Lung Development and Remodelling, Max Planck Institute for Heart and Lung Research, Bad Nauheim, Germany. ${ }^{3}$ Cardiovascular Research Center, Massachusetts General Hospital, Charlestown, MA, USA.
\end{abstract}

Correspondence: Rory E. Morty, Dept of Lung Development and Remodelling, Max Planck Institute for Heart and Lung Research, Parkstrasse 1, D-61231 Bad Nauheim, Germany. E-mail: rory.mortylampi-bn.mpg.de

ABSTRACT Aberrant remodelling of the extracellular matrix in the developing lung may underlie arrested alveolarisation associated with bronchopulmonary dysplasia (BPD). Transglutaminases are regulators of extracellular matrix remodelling. Therefore, the expression and activity of transglutaminases were assessed in lungs from human neonates with BPD and in a rodent model of BPD.

Transglutaminase expression and localisation were assessed by RT-PCR, immunoblotting, activity assay and immunohistochemical analyses of human and mouse lung tissues. Transglutaminase regulation by transforming growth factor (TGF)- $\beta$ was investigated in lung cells by luciferase-based reporter assay and RT-PCR. TGF- $\beta$ signalling was neutralised in vivo in an animal model of BPD, to determine whether TGF- $\beta$ mediated the hyperoxia-induced changes in transglutaminase expression.

Transglutaminase 2 expression was upregulated in the lungs of preterm infants with BPD and in the lungs of hyperoxia-exposed mouse pups, where lung development was arrested. Transglutaminase 2 localised to the developing alveolar septa. TGF- $\beta$ was identified as a regulator of transglutaminase 2 expression in human and mouse lung epithelial cells. In vivo neutralisation of TGF- $\beta$ signalling partially restored normal lung structure and normalised lung transglutaminase 2 mRNA expression.

Our data point to a role for perturbed transglutaminase 2 activity in the arrested alveolarisation associated with BPD.

○

@ERSpublications

Transglutaminase 2 may be a novel pathogenic factor in arrested lung development associated with BPD http://ow.ly/t7kMi

This article has supplementary material available from www.erj.ersjournals.com

Received: May 012013 | Accepted after revision: Jan 122014 | First published online: March 062014

Support statement: This study was supported by the German Research Foundation through grant Mo 1789/1 (to R.E. Morty), Excellence Cluster 147 "Cardio-Pulmonary System" (to S. Herold, K. Mayer, I. Vadász, W. Seeger and R.E. Morty), the Federal Ministry of Higher Education, Research and the Arts of the State of Hessen LOEWE Program (to S. Herold, K. Mayer, I. Vadász, W. Seeger and R.E. Morty), the German Center for Lung Research (Deutsches Zentrum für Lungenforschung; to T.J. Witsch, G. Niess, E. Sakkas, T. Likhoshvay, S. Becker, S. Herold, K. Mayer, I. Vadász, W. Seeger and R.E. Morty), and the US National Heart, Lung, and Blood Institute grant HL-094608 (to J.D. Roberts Jr).

Conflict of interest: None declared.

Copyright (OERS 2014 


\section{Introduction}

Premature infants frequently receive oxygen supplementation and mechanical ventilation as early lifesupport measures that, while effective, also cause bronchopulmonary dysplasia (BPD), a commonly encountered cause of morbidity and mortality in a neonatal intensive care setting [1]. While BPD is currently diagnosed exclusively by oxygen dependence criteria, the hallmark pathophysiological characteristic of BPD is an arrest of alveolarisation in the developing lung, which has long-term consequences for survivors. The pathophysiological basis of the arrested alveolarisation is not understood; however, perturbations of the remodelling and maturation of the extracellular matrix (ECM) are key features of BPD pathology [2]. Malformed collagen and elastin structures have been reported in the lungs of patients with BPD [3] and in animal models of BPD [4, 5]. How these ECM structures are malformed is not understood; however, one line of thinking is that the ECM cross-linking systems operative in affected lungs are deregulated, generating ECM structures that are consequently aberrantly remodelled, or that cannot be remodelled, thereby impeding the development of the immature lung $[5,6]$. Supporting this idea, increased expression and activity of lysyl oxidases, which are key ECM cross-linking enzymes, have been reported in clinical BPD and experimental animal models of BPD [6]. In the present study, we have addressed the expression and activity of a second family of protein cross-linking enzymes, the transglutaminases, in BPD.

Transglutaminases (EC 2.3.2.12) are $\mathrm{Ca}^{2+}$-dependent enzymes that catalyse the formation of isopeptide bonds either within or between polypeptide chains [7]. The transglutaminase family currently consists of nine members, including transglutaminases 1-7 (encoded by the TGM1-7 genes in humans and $\operatorname{tgm} 1-7$ genes in mice), and the related factor XIIIa and erythrocyte band 4.2 [7, 8]. The predominant transglutaminase catalytic product is a $\gamma$-glutamyl- $\varepsilon$-lysine cross-link formed by a transamidation reaction between a glutamyl residue $\gamma$-carboxamide group and a lysyl residue $\varepsilon$-amino group [7]. Transglutaminases are called "nature's biological glues" as the $\gamma$-glutamyl- $\varepsilon$-lysine cross-link is stable to proteolysis and mechanical stress [8]. Substrates of transglutaminases include the ECM proteins collagen II, V, VII and XI, the collagen III propeptide, and fibronectin [7, 8]. Given that transglutaminases can modify and cross-link many ECM proteins, and that pulmonary ECM remodelling and maturation are perturbed in clinical BPD and in experimental animal models of $\mathrm{BPD}$, transglutaminases appear to be interesting potential players in BPD pathogenesis. This idea is supported by two recent reports implicating transglutaminase 2 in pulmonary fibrosis $[9,10]$. For these reasons, we set out to examine the pulmonary expression, localisation and regulation of transglutaminases in clinical BPD and in an experimental animal model of BPD.

\section{Methods}

Human patient material

The use of human tissues in this study was approved by the Ethik-Kommission (the equivalent of an institutional review board in Germany) of the University of Giessen School of Medicine (Giessen, Germany) under approval number 189/09. Tissue harvesting from pre-term and term neonates has been described in detail [6]. Clinical data for patient material are provided in table 1.

\section{Animal model}

Animal experiments performed in Germany were approved by the Regierungspräsidium Gießen (the institutional animal care and use committee equivalent in Germany) under approval 22/2000. The hyperoxia-based animal model of BPD has been described by our group previously [6, 11], where hyperoxia-exposed pups develop a pronounced arrest of alveolarisation that was apparent within 5 days. Briefly, mouse pups were randomised to two groups within $12 \mathrm{~h}$ of birth (post-natal day (P)0.5), and exposed to $21 \%$ oxygen (normoxia) or $85 \%$ oxygen (hyperoxia).

\section{Cells and transforming growth factor $-\beta$ stimulation}

Human lung epithelial A549 (CCL-185) and H441 (HTB-174) cells were obtained commercially from the American Type Culture Collection (Manassas, VA, USA). Primary mouse lung fibroblasts and alveolar type II cells were isolated from adult $\mathrm{C} 57 \mathrm{Bl} / 6 \mathrm{~J}$ mouse lungs $[6,11]$. Primary human lung microvascular endothelial cells (C-12281), pulmonary artery smooth muscle cells (C-12521)) and lung fibroblasts (C-12360) were purchased from PromoCell (Heidelberg, Germany). For transforming growth factor (TGF)- $\beta$ stimulation, cells were exposed to TGF- $\beta_{1}\left(2 \mathrm{ng} \cdot \mathrm{mL}^{-1}\right.$ final concentration; R\&D Systems, Wiesbaden-Nordenstadt, Germany) for $18 \mathrm{~h}$. This represents a dose well within the standard range $\left(0.2-10 \mathrm{ng} \cdot \mathrm{mL}^{-1}\right)$ for in vitro TGF- $\beta_{1}$ stimulation studies $[6,11]$.

\section{Analysis of gene and protein expression}

Real-time RT-PCR was undertaken exactly as described previously $[6,11,12]$ using primers listed in online supplementary table $S 1$, all of which generated threshold cycle values $<35$ (fig. S2) for human (see table 1 
for number of subjects per group), mouse ( $n=5$ per group) or cell culture ( $n=3$ per group) material. The cDNA was synthesised as described previously $[6,11,12]$ from total RNA pools prepared from lung tissue or cultured cell homogenates. Immunoblotting was performed exactly as previously described $[6,11,12]$ using: goat anti-Tgm1 (diluted 1:200; antibody SC-18127; Santa Cruz Biotechnology, Santa Cruz, CA, USA); goat anti-Tgm2 (1:1000; 06-471; Upstate Biotechnology, Lake Placid, NY, USA); and rabbit anti- $\alpha$ tubulin (1:2500; SC-5286; Santa Cruz Biotechnology). Immune complexes were detected ( $\mathrm{n}=3$ per group per time-point) with donkey anti-goat IgG-horseradish peroxidase conjugate (1:1000; SC-2020; Santa Cruz Biotechnology) and goat anti-rabbit IgG-horseradish peroxidase conjugate (1:3000; 31460; Pierce, Bonn, Germany) using chemiluminescence.

\section{Immunohistochemistry}

Mouse lungs were pressure-fixed at $20 \mathrm{cmH}_{2} \mathrm{O}$ pressure and embedded in paraffin. Paraffin-embedded human and mouse lung tissue was sectioned at $3 \mu \mathrm{m}$. Transglutaminases or the $\gamma$-glutamyl- $\varepsilon$-lysine crosslink were detected in $3-\mu \mathrm{m}$ sections as previously described $[6,11,12]$ with goat anti-Tgm1, goat anti-Tgm2 or mouse anti-N- $\gamma$-glutamyl- $\varepsilon$-lysine (1:100; ab424; Abcam, Cambridge, UK) primary antibodies. Staining specificity was confirmed by the pre-adsorption of primary antibodies with a 100 -fold molar excess of a competing peptide (SC-50062P; Santa Cruz Biotechnology) for Tgm1 and H- $\gamma$-glutamyl- $\varepsilon$-lysyl-OH (G-1970; Bachem, Bubendorf, Switzerland) for the $\gamma$-glutamyl- $\varepsilon$-lysine cross-link. No pre-adsorption agent was available for Tgm2 detection, where isotype-matched, nonimmune antibodies substituted the specific antibody. Target antigen localisation was visualised with biotinylated rabbit anti-mouse (95-6543B; Invitrogen, Carlsbad, CA, USA) and anti-goat (A10518; Invitrogen) antibodies coupled with a streptavidinhorseradish peroxidase complex colorimetric detection system. Histological sections presented are representative of the staining patterns seen in at least three samples per group.

\section{Dual luciferase reporter assay}

The dual luciferase reporter assay to assess transglutaminase promoter induction by the TGF- $\beta$ signalling pathway was performed exactly as described previously $[6,11]$, using either the 2.2-kb human TGM1 [13] or the 4-kb mouse tgm2 [14] promoter to drive firefly luciferase expression from a pGL3 backbone in A549 or $\mathrm{H} 441$ cells ( $\mathrm{n}=5$ per group). Renilla luciferase activity, driven by the herpes simplex virus thymidine kinase promoter in pRLTK (Promega, Madison, WI, USA), yielded low to moderate levels of constitutive expression in cotransfected cells and was used to normalise firefly luciferase activity in a dual luciferase ratio.

\section{In vivo TGF- $\beta$ neutralisation experiments}

The inhibition of TGF- $\beta$ signalling in mouse pups in the hyperoxia-based animal model of BPD has shown by our own $[6,15]$ and other $[16,17]$ groups to normalise TGF- $\beta$ signalling, and to partially restore normal alveolar development. Neutralisation of TGF- $\beta$ signalling in mouse pup lungs ( $\mathrm{n}=6$ per group) was undertaken exactly as described previously using pan-TGF- $\beta$ neutralising IgG (1D11; R\&D Systems) and an isotype-matched nonimmune IgG (MOPC21; Sigma, St Louis, MO, USA) [6, 15].

\section{Transglutaminase 2 assay}

Transglutaminase 2 activity was assessed with a commercially available kit (CS1070; Sigma) with $100 \mu \mathrm{g}$ protein ( $n=7$ per group) over a 60 -min period. This assay determines transglutaminase 2 activity by measuring the covalent incorporation of the transglutaminase 2-specific substrate biotin-TVQQEL-OH into poly-L-lysine that has been immobilised on an ELISA plate. The biotin-TVQQEL-OH serves as the acyl donor, where conjugation occurs via the $\gamma$-carboxamide group of biotin-TVQQEL-OH. The incorporated biotin-TVQQEL-OH was assessed via biotin recognition of a streptavidin-peroxidase conjugate, detected using a spectrophotometer at $450 \mathrm{~nm}$ with $3,3^{\prime}, 5,5^{\prime}$-tetramethylbenzidine as a substrate.

\section{Statistics}

Data from patient samples were compared using one-way ANOVA with a Newman-Keuls post hoc test. For cell-culture studies, data were compared between two groups using an unpaired t-test, while groups containing more than two samples were compared using a one-way ANOVA with Tukey's post hoc test. Statistical outliers were determined using Grubbs' test for outliers.

\section{Results}

Transglutaminase expression is altered in the lungs of patients with or at risk for BPD

The expression of seven transglutaminases (TGM1-TGM7) was assessed in the lungs of seven patients with or at risk of BPD. Five patients had clinically defined BPD under current definition criteria. BPD groups are difficult to control for, as neonates are born prematurely and prematurely born neonates breathe gaseous air over a period where controls age-matched for gestational age continue to develop in utero with fluid-filled lungs. 


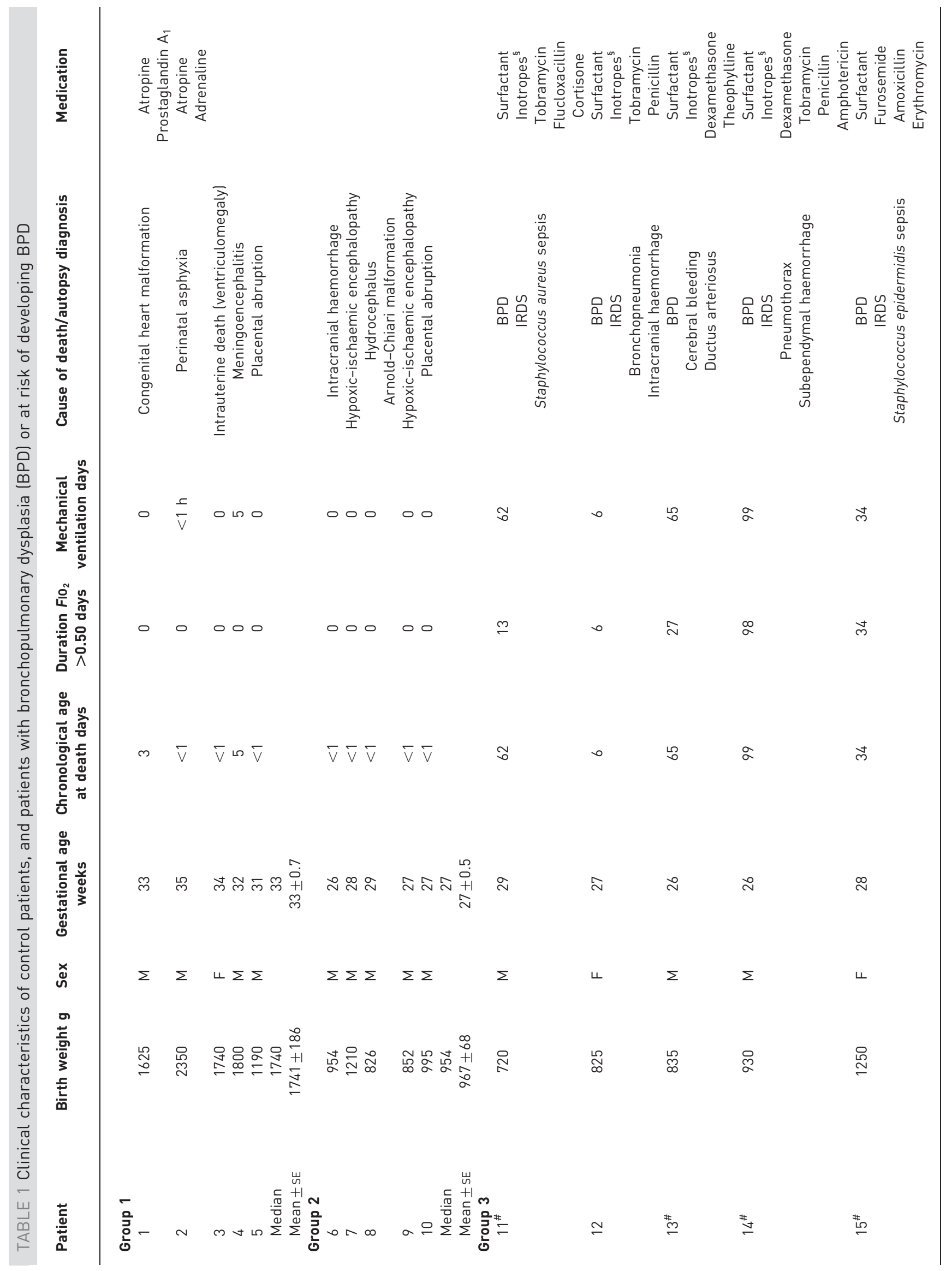




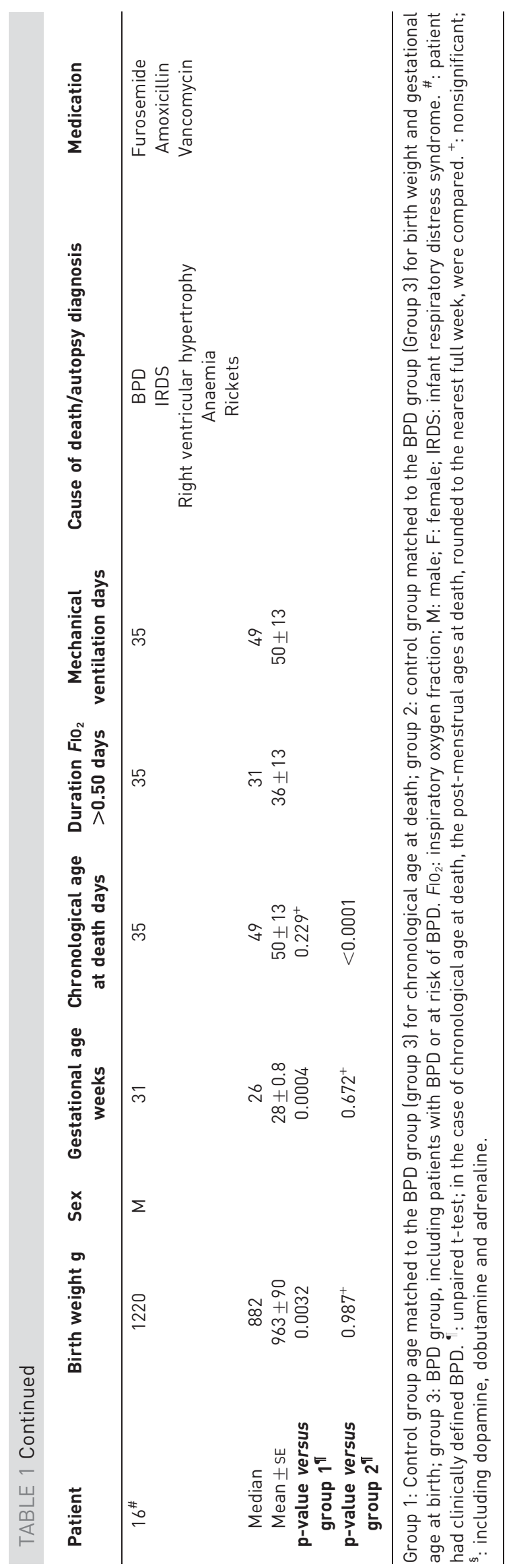



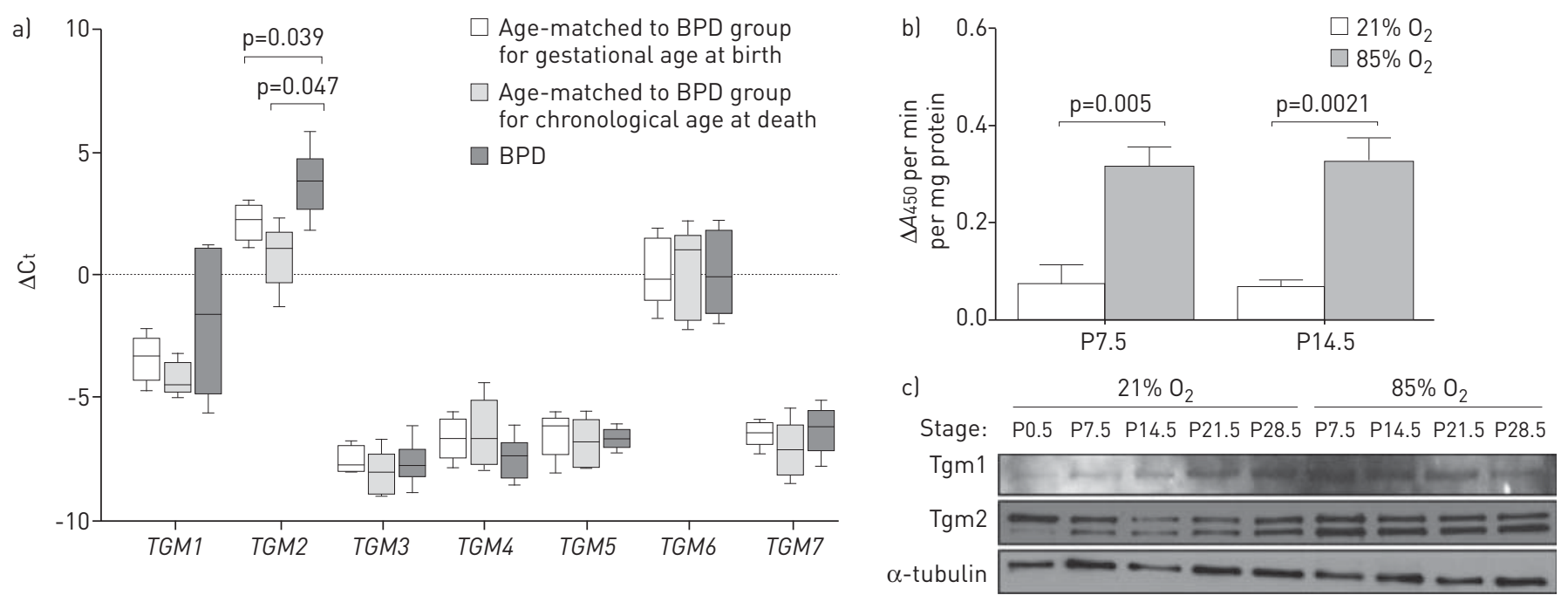

FIGURE 1 Lung transglutaminase expression in human neonates and mouse pups with normal and aberrant late lung development. a) The mRNA expression levels of all seven classical members (TGM1-TGM7) of the transglutaminase family were assessed by real-time RT-PCR with the primers listed in table S1, using mRNA pools from patients afflicted with or at risk of bronchopulmonary dysplasia (BPD), as well as mRNA pools from control patients matched to the BPD group either for gestational age at birth or for chronological age at death. Clinical characteristics of the patients are provided in table 1. The HPRT gene (hypoxanthine-guanine phosphoribosyltransferase) was used as a reference. The whiskers in the graph represent the range of the data points, the boxes represent the lower and upper quartiles, and the solid line within the quartile box indicates the median. All data sets were screened by Grubbs' test for significant outliers but none were found. p-values were assessed by one-way ANOVA with the Newman-Keuls post hoc test. b) Transglutaminase 2 activity was assessed by the incorporation of biotin-TVQQEL-OH into immobilised poly-L-lysine via the $\gamma$-carboxamide group of biotin-TVQQEL-OH. Data are presented as mean \pm SD Tgm 2 conjugating activity in mouse lung extracts. p-values were assessed by unpaired t-test ( $\mathrm{n}=7$ per group). c) Protein expression of Tgm 1 and Tgm 2 was assessed in protein extracts from whole-lung homogenates from mouse pups over the course of late lung development, during exposure to $21 \%$ or $85 \%$ oxygen from post-natal day $(\mathrm{P}) 0.5$, by immunoblotting. A single representative series is illustrated that is representative of at least two other series, which are quantified by densitometry in figure S1. $\Delta$ : change in; Ct: threshold cycle; A450: absorbance at $450 \mathrm{~nm}$.

Therefore, transglutaminase gene expression was assessed in two groups of control patients, one group matched to the BPD group for chronological age at death, while the other control group was matched to the BPD group for gestational age at birth. Only TGM2 expression was affected in the lungs of neonates with or at risk of $\mathrm{BPD}$, compared with infants age-matched for both gestational age at birth and chronological age at death (fig. 1a). Infants with or at risk of BPD also exhibited an increased range of mRNA expression levels for TGM1, compared with both control groups. However, the expression levels of TGM1 mRNA were not altered compared to either control group, taking $\mathrm{p}=0.05$ as a threshold value (fig. 1a).

\section{Lung transglutaminase expression is altered in an experimental animal model of BPD}

The expression of all seven members of the transglutaminases was assessed in normally developing mouse pups over the first month of post-natal lung development, as well as in the lungs of mouse pups exposed to normobaric hyperoxia ( $85 \%$ oxygen), where a pronounced arrest of alveolarisation has been noted $[6,11,17,18]$. In line with the findings in human BPD patients, transglutaminase 2 activity was also increased in aberrantly developing lungs from hyperoxia-exposed mouse pups at P7.5 and P14.5 (fig. 1b). Consistent with this observation, immunoblot analysis of Tgm 1 and Tgm 2 expression in the lungs of mouse pups over the first month of post-natal life, by which time lung development is complete, revealed a pronounced increase in protein abundance of Tgm 1 and Tgm 2 in hyperoxia-exposed mouse pups at P7.5 and P14.5 (fig. 1c, quantified in fig. S1). Particularly noteworthy was a sustained increase in protein abundance of Tgm2, which was correctly detected as a doublet, in the lungs of hyperoxia-exposed mouse pups at P7.5, P14.5 and P21.5, compared with littermates exposed to $21 \%$ oxygen (fig. 1c, quantified in fig. S1b). These data indicate that, similar to human patients with or at risk for BPD, the lung expression of Tgm2 is elevated in mouse pups in an experimental animal model of BPD. These observations prompted a screen of transglutaminase gene expression over the first 2 weeks of life, which is the critical secondary septation period. As early as day P2.5 (after 2 days of exposure to 85\% oxygen), elevated expression of $\operatorname{tgm} 1$ was observed (fig. 2). Over the first 14 days of life, hyperoxia-exposed mouse pups exhibited stably elevated tgm1 expression, in contrast to normoxia-exposed pups, where a dynamic expression pattern was evident, reaching a trough at P9.5, perhaps reflecting the normal developmental expression of (and thus changing need for) Tgm1 (fig. 2a). By P9.5, by which time alveolarisation is well underway, the expression of tgm1 

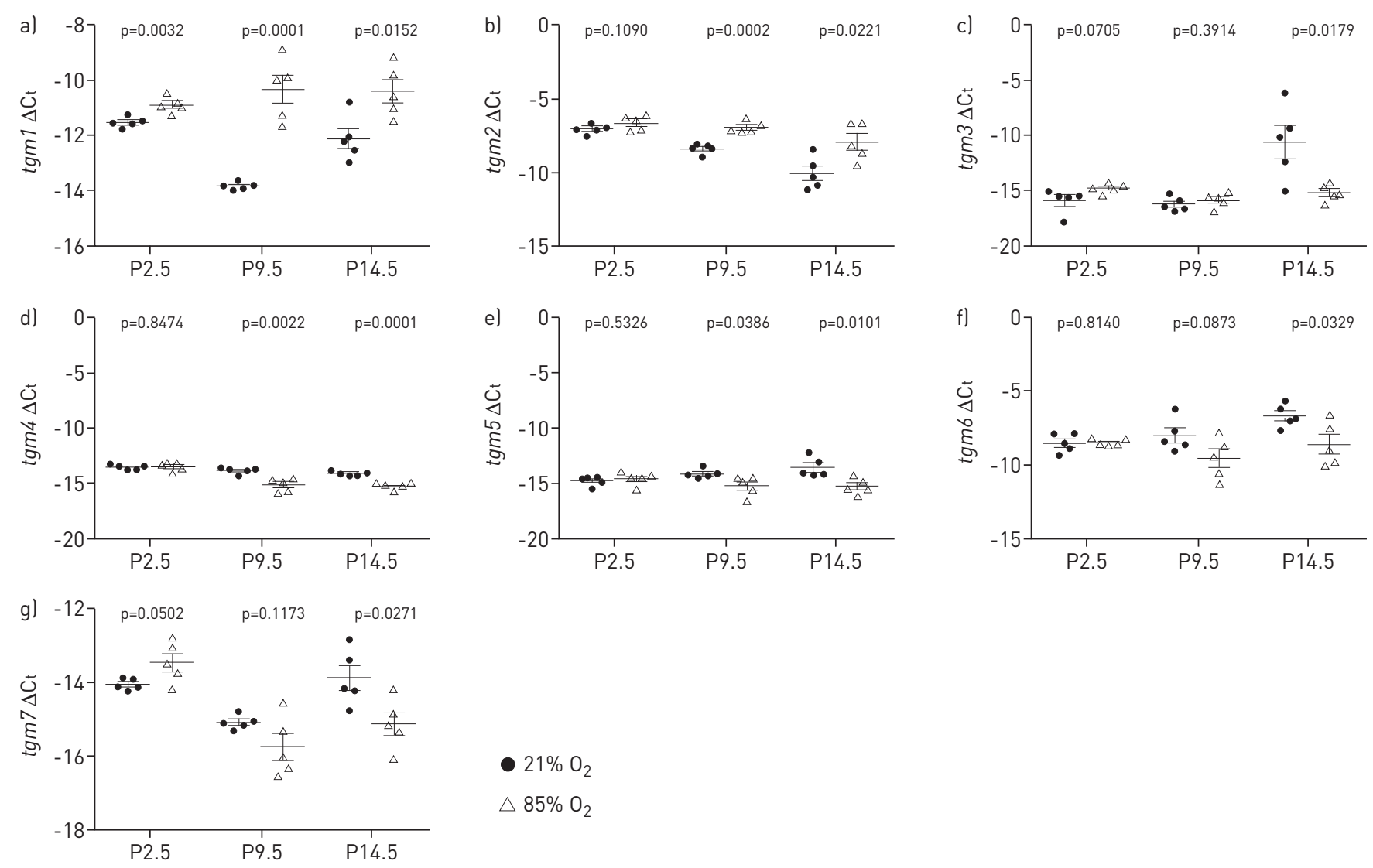

FIGURE 2 Expression of transglutaminase genes in the lungs of neonatal mouse pups with normal or aberrant late lung development. The mRNA expression levels of all seven classical members of the transglutaminase family of protein cross-linking enzymes were assessed by real-time RT-PCR with the primers listed in table S1, using mRNA pools from lung homogenates from mouse pups at post-natal day (P) 2.5, P9.5 and P14.5, after exposure to $21 \%$ or $85 \%$ oxygen from P0.5. a) $\operatorname{tgm} 1$; b) $\operatorname{tg} m 2$; c) $\operatorname{tgm} 3$; d) $\operatorname{tg} m 4$; e) $\operatorname{tgm} 5$; f) $\operatorname{tg} m 6$; g) $\operatorname{tg} m 7$. The $18 \mathrm{~S}$ ribosomal RNA was used as a reference. Data are presented as mean $\pm \mathrm{SE}(\mathrm{n}=5)$. p-values compared the $21 \%$ and $85 \%$ oxygen groups, and were assessed by one-way ANOVA with Tukey's post hoc test. $\Delta$ : change in; Ct: threshold cycle.

and $\operatorname{tgm} 2$ was elevated in the lungs of hyperoxia-exposed mouse pups, while expression of $\operatorname{tgm} 4$ and $\operatorname{tgm} 5$ was down-regulated (fig. 2).

\section{Transglutaminases and transglutaminase activity localise to regions of intense remodelling during alveolarisation}

Transglutaminase activity, assessed by the presence of the transglutaminase-generated $\gamma$-glutamyl- $\varepsilon$-lysine cross-link, was detected in the developing mouse lungs (fig. 3), where staining was noted in the developing septa and epithelial structures of the parenchyma. A parallel pattern of expression was also noted for both Tgm1 (fig. 3f-i) and Tgm2 (fig. 3k-n). Furthermore, Tgm2 staining could be seen in the endothelial lining of large vessels and in regions of the vessel wall (fig. $3 \mathrm{~h}$ ). While the authors do not consider the relative intensity of immunohistochemical staining to be quantitative, the apparent increase in staining intensity for Tgm 2 in the parenchymal regions of lungs from mouse pups exposed to $85 \%$ oxygen (fig. $3 \mathrm{mn}$ ), compared with comparatively less Tgm 2 staining in the lungs of $21 \%$ oxygen-exposed littermates (fig. $3 \mathrm{k}-1$ ), was consistent with the Tgm2 immunoblot data (fig. 1c), which revealed an increased lung Tgm2 expression after exposure to hyperoxia.

\section{Transglutaminase expression is regulated by TGF- $\beta_{1}$}

The regulation of transglutaminase expression by TGF- $\beta_{1}$ was assessed in the constituent cell types of the developing alveolus. While TGF- $\beta_{1}$ did not impact TGM1 expression in the human A549 cell-line (fig. 4a), TGF- $\beta_{1}$ did upregulate mRNA levels of $\operatorname{tgm} 1$ in primary mouse alveolar type II cells (fig. $4 \mathrm{~b}$ ) and upregulated expression of TGM2 in A549 cells (fig. 4e) and tgm2 in primary mouse alveolar type II cells (fig. 4f). TGF- $\beta_{1}$ was without effect on TGM1 (fig. 4c) or TGM2 (fig. 4g) expression in primary human lung fibroblasts, or on $\operatorname{tgm} 1$ (fig. $4 \mathrm{~d}$ ) or $\operatorname{tgm} 2$ (fig. 4h) expression in primary mouse lung fibroblasts. The impact of TGF- $\beta_{1}$ was also assessed for all other transglutaminases, where the only notable effects of TGF- $\beta_{1}$ were the downregulation of TGM3 mRNA levels in A549 cells (fig. S3) and a downregulation of TGM7 


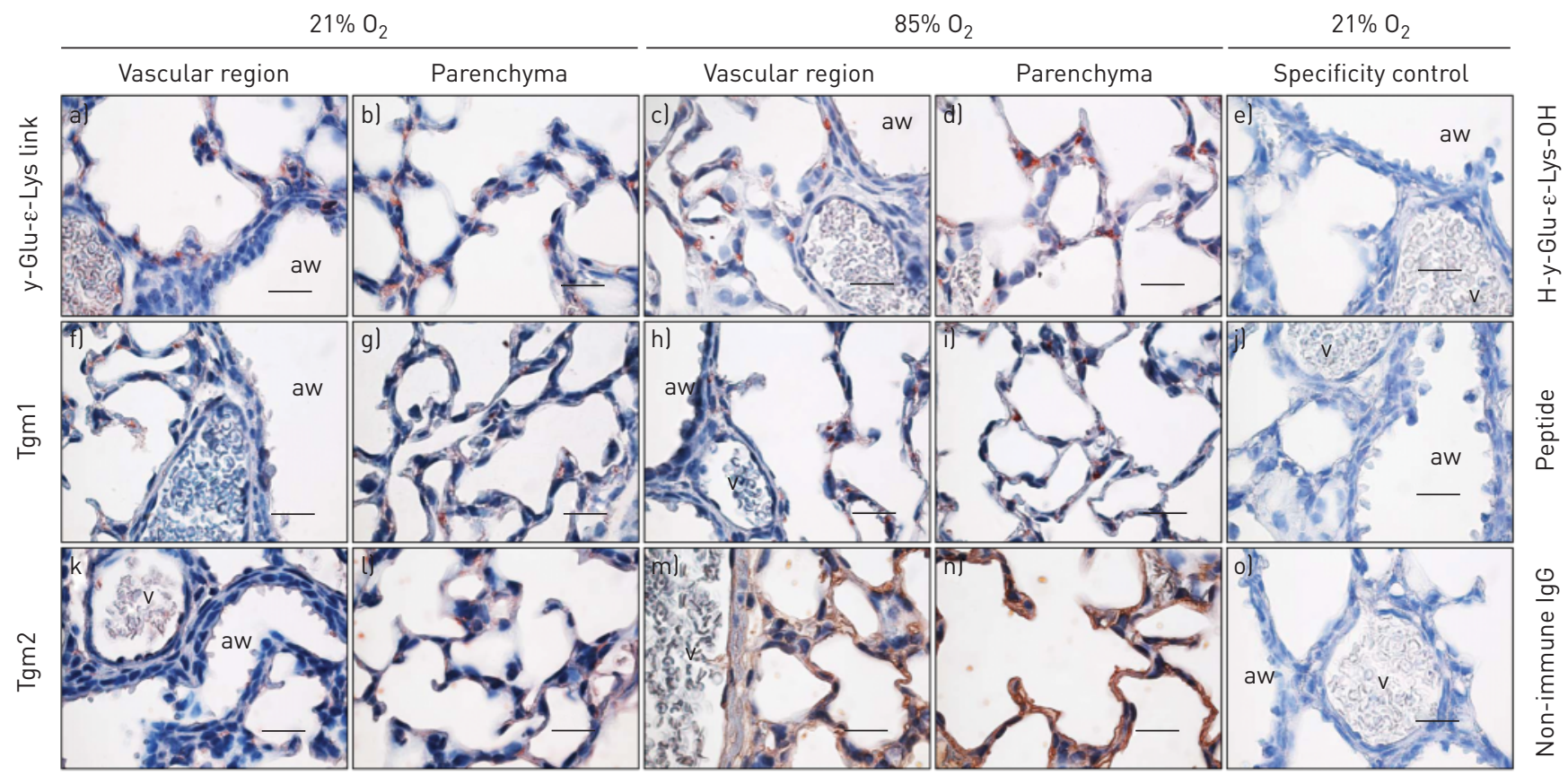

FIGURE 3 Localisation of transglutaminase expression in the parenchyma and vascular regions of the lungs of mouse pups at post-natal day (P)7.5, after exposure to $21 \%$ or $85 \%$ oxygen from P0.5. e, $\mathrm{j}, \mathrm{o}$ ) The specificity control column of photomicrographs indicates staining of the parenchyma and vascular regions of the lungs of mouse pups at P7.5, after exposure to $21 \%$ oxygen from P0.5. For specificity control, primary antibodies against the $\gamma$-glutamyl- $\varepsilon$-lysine cross-link and Tgm 1 were pre-adsorbed with the H- $\gamma$-glutamyl- $\varepsilon$-lysyl-OH dipeptide or the Tgm1 immunogenic peptide, respectively. In the case of Tgm2, primary antibodies were replaced with nonimmune, isotype-matched control IgG, as no pre-adsorption agent is available for anti-Tgm2 antibodies. The airways (aw) and vessels (v) are indicated. Scale bars $=20 \mu \mathrm{m}$.

mRNA levels in primary human lung microvascular endothelial cells (fig. S4a). Interestingly, the downregulation of TGM3 and TGM7 expression by TGF- $\beta_{1}$ correlated with the downregulation of $\operatorname{tgm} 3$ and $\operatorname{tgm} 7$ in the mouse model of BPD (fig. 2), suggesting that TGF- $\beta_{1}$ may mediate this phenomenon in hyperoxia-exposed mouse pups. There was no impact of TGF- $\beta_{1}$ on the expression levels of other tgm genes in primary mouse alveolar type II cells (fig. S5a) or in primary mouse lung fibroblasts (fig. S5b).

\section{TGF- $\beta$ signalling drives transglutaminase promoter activation in lung epithelial cells}

The human TGM1 promoter was not activated by stimulation of A549 cells with TGF- $\beta_{1}$ (fig. $5 \mathrm{a}$ ); however, TGF- $\beta_{1}$ stimulation did activate the TGM1 promoter in H441 cells (fig. 5a). Furthermore, the mouse tgm 2 promoter was activated by TGF- $\beta_{1}$ stimulation of both A549 (fig. 5c) and H441 (fig. 5d) cells. These data indicate that TGF- $\beta_{1}$ can be a regulator of transglutaminase expression in the lung epithelium and are supported by the mRNA expression data in A549 cells and primary mouse alveolar type II cells (fig. 4).

\section{Hyperoxia-induced changes in lung Tgm2 expression are mediated by TGF- $\beta_{1}$}

Neutralisation of TGF- $\beta$ signalling in animal models of arrested lung development both normalises lung TGF- $\beta$ signalling and partially restores normal alveolarisation [17-19]. In the present study, it was revealed that in vivo neutralisation of TGF- $\beta$ signalling has no impact on hyperoxia-induced increases in $\operatorname{tgm} 1$ expression (fig. 6a). However, neutralisation of TGF- $\beta$ signalling with the 1 D11 antibody did normalise $\operatorname{tgm} 2$ expression in the lungs of hyperoxia-exposed mouse pups. While the authors do not consider the intensity of immunohistochemical staining to be quantitative, the apparent increase in staining intensity for Tgm 2 in the developing septa of lungs from mouse pups exposed to $85 \%$ oxygen (fig. S6c), contrasted with comparatively less Tgm 2 staining in the lungs of $21 \%$ oxygen-exposed littermates (fig. S6a), appeared to be diminished when compared with developing septa of lungs from mouse pups exposed to $85 \%$ oxygen that received a TGF- $\beta$-neutralising antibody (fig. S6d versus S6c).

\section{Transglutaminase 2 is widely expressed in the lungs of human neonates}

Given the increased mRNA expression of TGM2 in the lungs of human patients with BPD, lung tissues from normally developing human lungs as well as lung tissues from BPD patients were examined for TGM2 localisation by immunohistochemistry (fig. 7). TGM2 immunoreactivity was observed in the alveolar septa, airway epithelium, and airway and vascular smooth muscle walls, as well as the endothelium. 

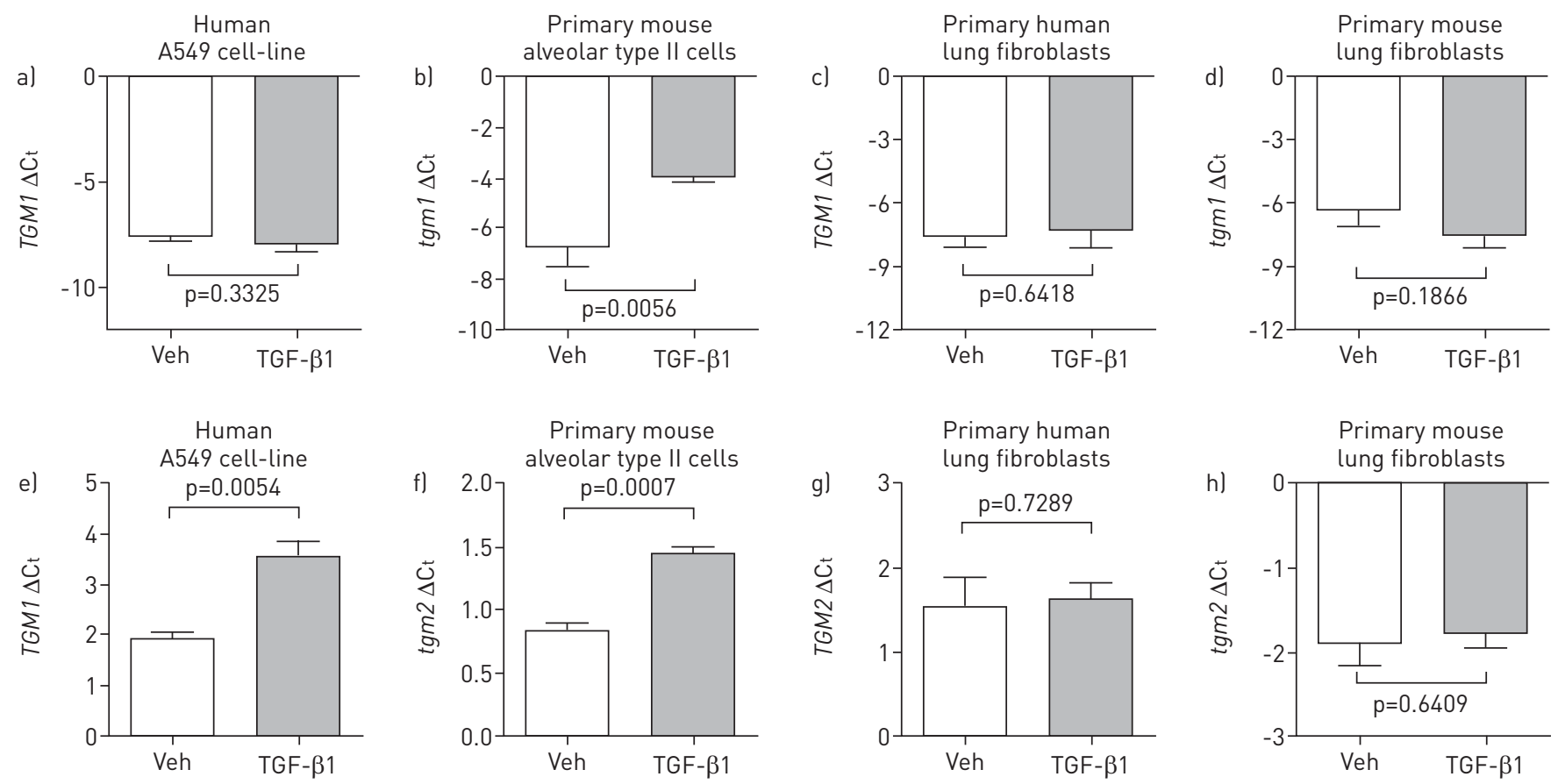

FIGURE 4 Regulation of transglutaminase mRNA levels by transforming growth factor (TGF)- $\beta_{1}$. The influence of TGF- $\beta_{1}$ on a-d) transglutaminase 1 and e-h) transglutaminase 2 gene expression was assessed in a, e) the human A549 lung epithelial cell-line, b, f) primary mouse alveolar type II cells, c, g) primary human lung fibroblasts and d, h) primary mouse lung fibroblasts. Cells were treated with vehicle (Veh) alone (PBS) or TGF- $\beta_{1}\left(2 \mathrm{ng} \cdot \mathrm{mL}^{-1} \mathrm{in} \mathrm{PBS}\right)$. The HPRT and $h r p t$ genes (hypoxanthine-guanine phosphoribosyltransferase) were employed as reference genes for human and mouse cells, respectively. Primer sequences are provided in table S1. The impact of TGF- $\beta_{1}$ on transglutaminase gene expression (TGM1-TGM7) in primary human lung vascular endothelial cells and primary human pulmonary artery smooth muscle cells, and for the remaining transglutaminase genes $(\operatorname{tg} m 3-\operatorname{tg} m 7)$ in primary mouse alveolar type II cells are provided in the online supplement in figures $\mathrm{S} 1$ and $\mathrm{S} 2$, respectively. $\mathrm{p}$-values were assessed by unpaired $\mathrm{t}$-test $\left(\mathrm{n}=3\right.$ per group) and compare Veh versus TGF- $\beta_{1}$-treated groups. $\Delta$ : change in; Ct: threshold cycle.

\section{Discussion}

Although transglutaminases have been widely studied in neurodegenerative and autoimmune diseases, and genetic diseases including lamellar ichthyosis and factor XIII deficiency [7], transglutaminases have received scant attention in the lung. Two recent reports have implicated Tgm2 in pulmonary fibrosis [9, 10], while one earlier study detected Tgm 2 in the normally developing rat lung, where externalisation of Tgm 2 from intracellular to extracellular pools was thought to delay basement membrane remodelling and to stabilise extracellular components such as microfibrils [20]. Our data reveal pronounced deregulation of transglutaminase 2 in clinical BPD and in an experimental animal model of BPD. The localisation studies reported here place transglutaminase 2 in the parenchyma of the developing mouse lung, a region of intense remodelling during alveolarisation. TGF- $\beta$ was identified as a regulator of epithelial tgm 2 expression and as the factor that mediated hyperoxia-induced changes in tgm 2 expression in an experimental animal model of BPD. While the bulk of these data point to perturbed TGM2 expression in clinical BPD, altered Tgm1 expression was also noted in the mouse BPD model. This is interesting, as Tgm1 has been reported to be expressed in the normal respiratory epithelium, as well as in bronchial pre-invasive lesions and lung cancer [21], and increased Tgm1 expression is associated with squamous metaplasia of the respiratory epithelium [22], which is in line with the squamous metaplasia noted in BPD.

Abnormally elevated transglutaminase 1 and 2 expression could promote aberrant late lung development. It has been proposed that ECM cross-linking enzymes, such at the lysyl oxidases, are abnormally active in clinical BPD and an experimental animal model of BPD [6]. The same appears to be true for transglutaminases, which may then drive excessive cross-linking (and, hence, excessive stabilisation) of the lung ECM, rendering the ECM resistant to proteolysis, thus "locking" the lung structure and preventing the remodelling processes that normally facilitate alveolarisation. Transglutaminases are particularly active in basement membrane remodelling and the final phase of late lung development is achieved by fusion of epithelial and endothelial basement membranes [20]. Improperly cross-linked basement membrane collagens and other structural molecules may hinder the prerequisite remodelling of basement membranes to promote basement membrane fusion. 

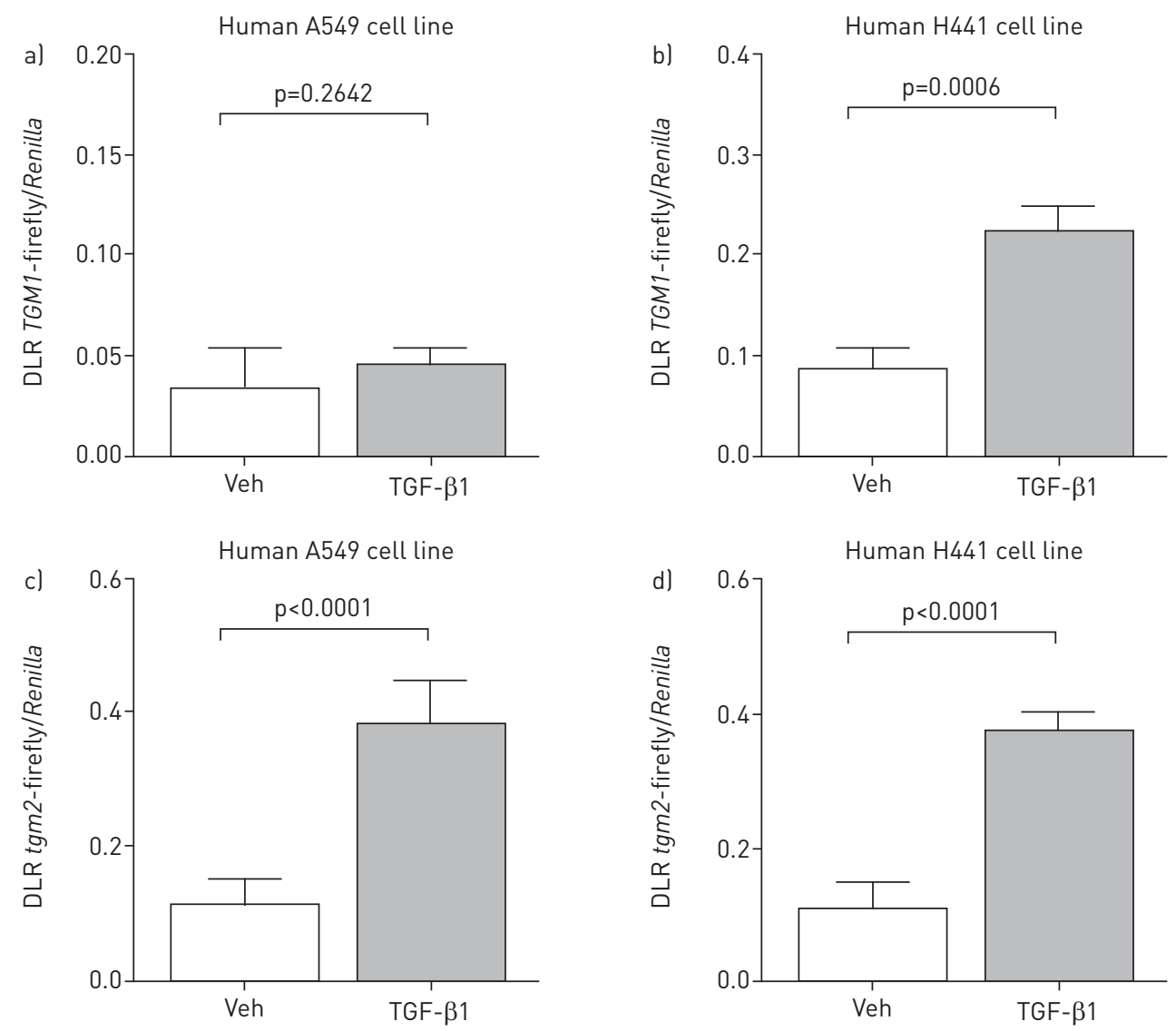

FIGURE 5 Regulation of transglutaminase promoter activity by transforming growth factor (TGF)- $\beta$ signalling in human epithelial cell lines. Following transfection with plasmid constructs, a, b) the activity of the human TGM1 and c, d) mouse tgm2 promoters was assessed by dual luciferase assay a, c) in the human A549 cell-line, as a model of the alveolar epithelium, and b, d) in the human H441 cell-line, as a model of the conducting airway epithelium. Transfected cells were stimulated with vehicle (Veh) $\left(4 \mathrm{mM}\right.$ hydrochloric acid, $1 \mathrm{mg} \cdot \mathrm{mL}^{-1}$ bovine serum albumin; diluted 1:500 in cell-culture medium) or TGF- $\beta_{1}$ alone ( $2 \mathrm{ng} \cdot \mathrm{mL}^{-1}$; in cell-culture medium). Data points reflect the dual luciferase ratio (DLR) in which transglutaminase-driven firefly luciferase is normalised to Renilla luciferase expression driven by the herpes simplex virus thymidine kinase promoter, providing low to moderate levels of constitutive expression in cotransfected mammalian cells. $\mathrm{p}$-values compare Veh- versus TGF- $\beta$-treated groups and were assessed by unpaired $\mathrm{t}$-test $(\mathrm{n}=5$ per group).

In addition to stabilising the ECM, transglutaminases have other notable functions that may be impacted in BPD. The epithelial lining of the lungs of infants with BPD is damaged by mechanical stretch and strain, and TGM2 is known to promote membrane resealing after mechanical damage to human lung A549 cells [23]. Along these lines, Tgm1 has also been shown to be critical for the structural integrity of mouse epithelial cells, by stabilising cadherin-based adherens junctions [24]. Thus, perturbed transglutaminase 1 and 2 expression may impact epithelial cell structural integrity and membrane repair, which are important processes in a premature, inflamed lung that is subjected to the stresses associated with mechanical ventilation and oxygen toxicity, which form part of the medical management of patients with BPD.

TGF- $\beta$ was identified in this study as a regulator of $\operatorname{tg} m 2$ expression in the mouse lung epithelium and as the mediator of hyperoxia-driven lung tgm 2 expression in the mouse BPD model. These data add to an emerging body of evidence that highlight TGF- $\beta$ as a mediator of both experimental $[12,17,18,25]$ and clinical [26] BPD, where it has been solidly established by our own and other groups that in vivo neutralisation of TGF- $\beta$ in hypoxia or hyperoxia models of BPD partially restores normal lung architecture. Our findings here demonstrate that in vivo TGF- $\beta$ neutralisation also restored normal $\operatorname{tg} m 2$ expression in the lungs of hyperoxia-treated mouse pups makes a strong case for Tgm2 as a pathogenic ECM remodelling factor in this rodent BPD model. This idea is further strengthened by our findings that TGM2 expression is elevated in human neonates with or at risk for BPD.

TGF- $\beta_{1}$ did not drive transglutaminase expression in lung fibroblasts, which contrasts with observations made in dermal fibroblasts [27]. However, OLSEN et al. [10] also noted that TGF- $\beta_{1}$ did not drive TGM2 

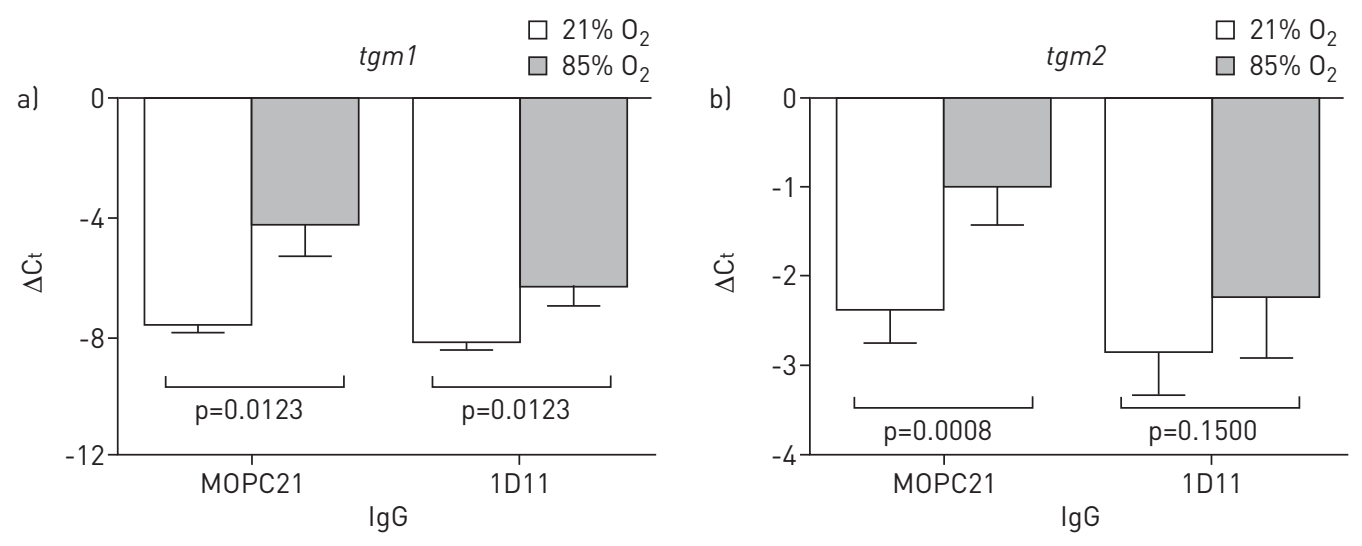

FIGURE 6 Impact of in vivo neutralisation of transforming growth factor (TGF)- $\beta$ signalling on hyperoxia-induced transglutaminase gene expression. Gene expression of mRNA encoding a) $\operatorname{tg} m 1$ and b) $\operatorname{tg} m 2$ was assessed in the lungs of mouse pups at post-natal day (P) 10.5 , which had inspired $21 \%$ or $85 \%$ oxygen from P0.5, and were treated with a panTGF- $\beta_{1-3}$-neutralising IgG (1D11) or with an isotype-matched control (MOPC21) IgG. The mRNA levels were assessed by real-time RT-PCR in mRNA pools from whole-lung homogenates, using the primers in table S1, with the $18 \mathrm{~S}$ ribosomal RNA serving as a reference. $\Delta$ : change in; Ct: threshold cycle. p-values were assessed by unpaired t-test, and were used to compare the $21 \%$ and $85 \%$ oxygen groups ( $n=6$ per group).

expression in human lung fibroblasts, although TGF- $\beta_{1}$ did rapidly promote the externalisation and secretion of TGM2 by human lung fibroblasts. Thus, in the context of BPD, it is likely that TGF- $\beta$ drives increased gene expression of transglutaminase II in epithelial cells, and also, increased secretion (and, hence, extracellular activity) of fibroblast-derived transglutaminase 2. In addition to ECM cross-linking functions, intracellular Tgm2 has also been credited with acting: 1) as a rheostat, regulating both apoptosis and autophagy in mouse fibroblasts [28]; and 2) in regulating adhesion [29] and migration [10] of mouse fibroblasts. Thus, perturbations to transglutaminase 2 expression may influence the spatiotemporal

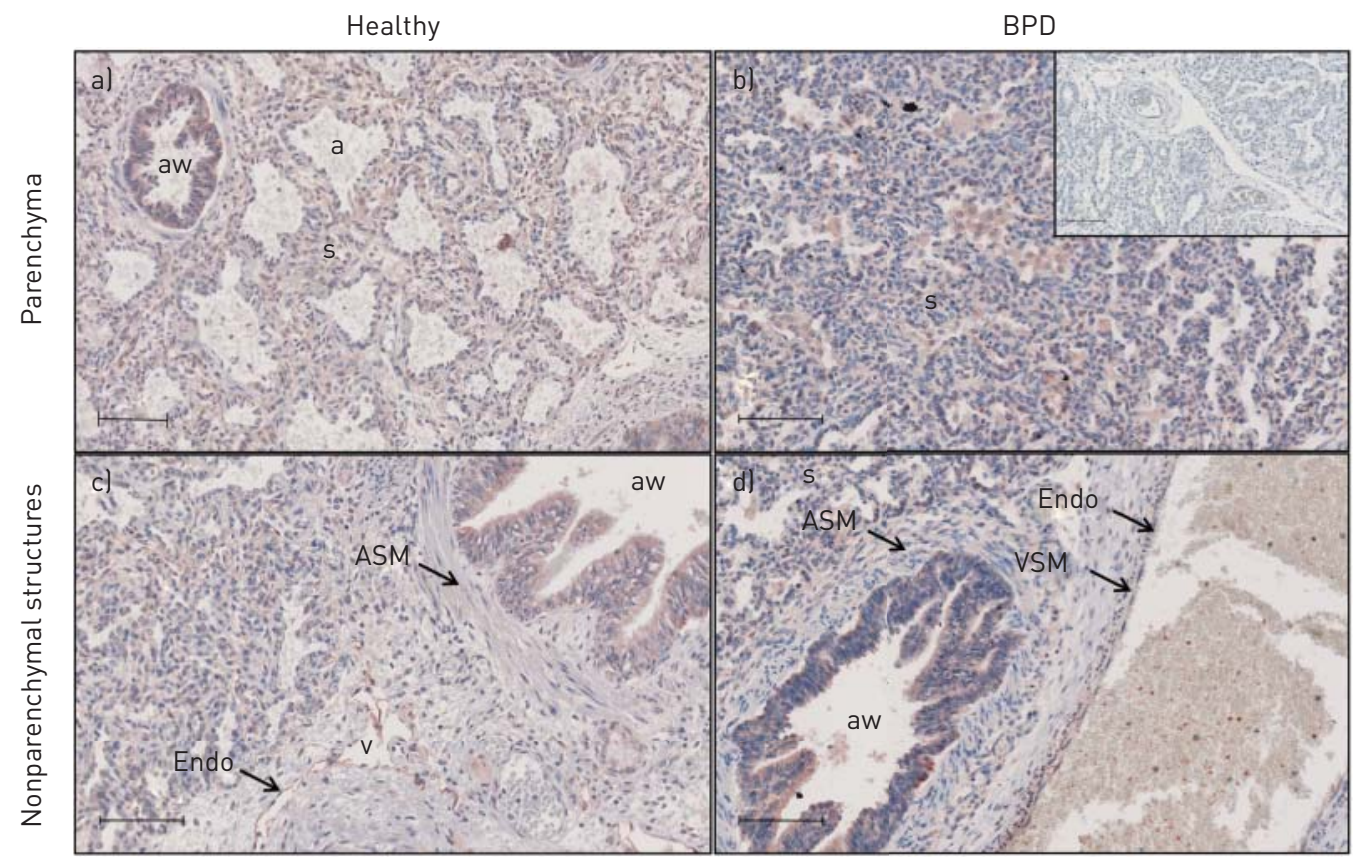

FIGURE 7 Transglutaminase 2 expression in the lung tissues of human neonates with normally or aberrantly developing lungs. The tissue localisation of TGM2 protein was assessed a, c) in the lung tissues of human neonates with normal lung development (patient 2 in table 1) and $\mathrm{b}, \mathrm{d}$ ) in the lung tissue of a patient with bronchopulmonary dysplasia (BPD) (patient 15 in table 1). c) As a specificity control, primary antibodies were replaced with nonimmune isotype-matched control IgG, as no pre-adsorption agent is available for anti-TGM2 antibodies (inset). The airways (aw), airway smooth muscle (ASM), alveolar airspaces (a), endothelium (Endo), vascular smooth muscle (VSM), alveolar septa (s) and vessels (v) are indicated. Scale bars $=100 \mu \mathrm{m}$. 
organisation of ECM deposition by fibroblasts in the developing alveolar septa. Interestingly, transglutaminase 2 is also known to activate latent TGF- $\beta$ to active TGF- $\beta$ [30], suggesting a positive feedback loop, where increased TGF- $\beta$ activity may drive increased Tgm 2 expression and activity, which, in turn, then escalates TGF- $\beta$ activity in the lung, leading to a vicious circle where Tgm 2 and TGF- $\beta$ drive each other's expression.

BPD is a complicated disease and it should be noted that the animal model employed only mimics the arrested septation component of the alveolar simplification seen in patients with BPD. Furthermore, the mouse model employed in the current study replicates the response of alveolar development to oxygeninduced lung injury, whereas the humans who develop BPD might also have pathological contributions to lung disease due to other factors (such as inflammation and volutrauma) in addition to hyperaemia. This may be regarded as a limitation of the model. However, it is also notable that term mouse pups are born at the same stage of lung development as are pre-term infants who develop BPD and, as such, the mouse model is more suitable for BPD studies than is generally appreciated. Additionally, alveolar simplification due to arrested secondary septation is the key histopathological characteristic of "new" BPD and this was modelled very well in the mouse pup model. Other limitations of this study include the use of cells derived from adult (not neonatal) humans and mice, which may impact the extrapolation of the in vitro results to the situation in the neonate. It should also be kept in mind that both control patient groups did not survive post-natally and, as such, no control group that was age-matched for post-natal age has been included. Thus, the impact of post-natal life alone on transglutaminase expression has not been estimated in this study.

In spite of these limitations, our data do make a strong case for a pathogenic role for a second family of protein cross-linking enzymes, the transglutaminases, in the arrested lung development associated with clinical BPD and experimental animal models of BPD. Follow-up studies with transglutaminase inhibitors, currently being initiated by the investigators' laboratories, will assess whether transglutaminases play a causal role or represent therapeutic targets, in experimental animal models of BPD.

\section{Acknowledgements}

The authors thank R. Bland (Stanford University, Stanford, CA, USA), T. Mariani (University of Rochester, Rochester, MN, USA), B. Mecham (Washington University School of Medicine, St Louis, MO, USA), R. Pierce (Washington University School of Medicine), I. Reiss (Erasmus Medical Centre, Rotterdam, The Netherlands), D. Tibboel (Erasmus Medical Centre) and D. Warburton (University of Southern California, Los Angeles, CA, USA) for constructive discussions and reagents. The pGL3-TGM1 and pGL3-tgm2 promoter constructs were kind gifts from B. Rice and L. Nagy, respectively.

\section{References}

Jobe AH, Bancalari E. Bronchopulmonary dysplasia. Am J Respir Crit Care Med 2001; 163: 1723-1729.

Madurga A, Mižíková I, Ruiz-Camp J, et al. Recent advances in late lung development and the pathogenesis of bronchopulmonary dysplasia. Am J Physiol Lung Cell Mol Physiol 2013; 305: L893-L905.

3 Thibeault DW, Mabry SM, Ekekezie, II, et al. Collagen scaffolding during development and its deformation with chronic lung disease. Pediatrics 2003; 111: 766-776.

Albertine KH, Jones GP, Starcher BC, et al. Chronic lung injury in preterm lambs. Disordered respiratory tract development. Am J Respir Crit Care Med 1999; 159: 945-958.

Bourbon J, Boucherat O, Chailley-Heu B, et al. Control mechanisms of lung alveolar development and their disorders in bronchopulmonary dysplasia. Pediatr Res 2005; 57: 38R-46R.

6 Kumarasamy A, Schmitt I, Nave AH, et al. Lysyl oxidase activity is dysregulated during impaired alveolarization of mouse and human lungs. Am J Respir Crit Care Med 2009; 180: 1239-1252.

7 Lorand L, Graham RM. Transglutaminases: crosslinking enzymes with pleiotropic functions. Nat Rev Mol Cell Biol 2003; 4: 140-156.

8 Griffin M, Casadio R, Bergamini CM. Transglutaminases: nature's biological glues. Biochem J 2002; 368: 377-396.

9 Oh K, Park HB, Byoun OJ, et al. Epithelial transglutaminase 2 is needed for T cell interleukin-17 production and subsequent pulmonary inflammation and fibrosis in bleomycin-treated mice. J Exp Med 2011; 208: 1707-1719.

10 Olsen KC, Sapinoro RE, Kottmann RM, et al. Transglutaminase 2 and its role in pulmonary fibrosis. Am J Respir Crit Care Med 2011; 184: 699-707.

11 Alejandre-Alcázar MA, Kwapiszewska G, Reiss I, et al. Hyperoxia modulates TGF-beta/BMP signaling in a mouse model of bronchopulmonary dysplasia. Am J Physiol Lung Cell Mol Physiol 2007; 292: L537-L549.

12 Alejandre-Alcázar MA, Michiels-Corsten M, Vicencio AG, et al. TGF- $\beta$ signaling is dynamically regulated during the alveolarization of rodent and human lungs. Dev Dyn 2008; 237: 259-269.

13 Jessen BA, Qin Q, Rice RH. Functional AP1 and CRE response elements in the human keratinocyte transglutaminase promoter mediating Whn suppression. Gene 2000; 254: 77-85.

14 Nagy L, Saydak M, Shipley N, et al. Identification and characterization of a versatile retinoid response element (retinoic acid receptor response element-retinoid $\mathrm{X}$ receptor response element) in the mouse tissue transglutaminase gene promoter. J Biol Chem 1996; 271: 4355-4365.

15 Nakanishi H, Sugiura T, Streisand JB, et al. TGF- $\beta$-neutralizing antibodies improve pulmonary alveologenesis and vasculogenesis in the injured newborn lung. Am J Physiol Lung Cell Mol Physiol 2007; 293: L151-L161.

16 Hilgendorff A, Parai K, Ertsey R, et al. Neonatal mice genetically modified to express the elastase inhibitor elafin are protected against the adverse effects of mechanical ventilation on lung growth. Am J Physiol Lung Cell Mol Physiol 2012; 303: L215-L227. 

inhibiting TGF- $\beta$ signaling. Am J Physiol Lung Cell Mol Physiol 2011; 301: L721-L730.

18 Nicola T, Ambalavanan N, Zhang W, et al. Hypoxia-induced inhibition of lung development is attenuated by the peroxisome proliferator-activated receptor-gamma agonist rosiglitazone. Am J Physiol Lung Cell Mol Physiol 2011; 301: L125-L134.

19 Olave N, Nicola T, Zhang W, et al. Transforming growth factor- $\beta$ regulates endothelin-1 signaling in the newborn mouse lung during hypoxia exposure. Am J Physiol Lung Cell Mol Physiol 2012; 302: L857-L865.

20 Schittny JC, Paulsson M, Vallan C, et al. Protein cross-linking mediated by tissue transglutaminase correlates with the maturation of extracellular matrices during lung development. Am J Respir Cell Mol Biol 1997; 17: 334-343.

21 Martinet N, Bonnard L, Regnault V, et al. In vivo transglutaminase type 1 expression in normal lung, preinvasive bronchial lesions, and lung cancer. Am J Respir Cell Mol Biol 2003; 28: 428-435.

22 Vollberg TM, George MD, Nervi C, et al. Regulation of type I and type II transglutaminase in normal human bronchial epithelial and lung carcinoma cells. Am J Respir Cell Mol Biol 1992; 7: 10-18.

23 Kawai Y, Wada F, Sugimura Y, et al. Transglutaminase 2 activity promotes membrane resealing after mechanical damage in the lung cancer cell line A549. Cell Biol Int 2008; 32: 928-934.

24 Hiiragi T, Sasaki H, Nagafuchi A, et al. Transglutaminase type 1 and its cross-linking activity are concentrated at adherens junctions in simple epithelial cells. J Biol Chem 1999; 274: 34148-34154.

25 Tarantal AF, Chen H, Shi TT, et al. Overexpression of transforming growth factor-betal in fetal monkey lung results in prenatal pulmonary fibrosis. Eur Respir J 2010; 36: 907-914.

26 Been JV, Debeer A, van Iwaarden JF, et al. Early alterations of growth factor patterns in bronchoalveolar lavage fluid from preterm infants developing bronchopulmonary dysplasia. Pediatr Res 2010; 67: 83-89.

27 Quan G, Choi JY, Lee DS, et al. TGF- $\beta 1$ up-regulates transglutaminase two and fibronectin in dermal fibroblasts: a possible mechanism for the stabilization of tissue inflammation. Arch Dermatol Res 2005; 297: 84-90.

28 Rossin F, D'Eletto M, Macdonald D, et al. TG2 transamidating activity acts as a reostat controlling the interplay between apoptosis and autophagy. Amino Acids 2012; 42: 1793-1802.

29 Gentile V, Thomazy V, Piacentini M, et al. Expression of tissue transglutaminase in Balb-C 3T3 fibroblasts: effects on cellular morphology and adhesion. J Cell Biol 1992; 119: 463-474.

30 Kojima S, Nara K, Rifkin DB. Requirement for transglutaminase in the activation of latent transforming growth factor- $\beta$ in bovine endothelial cells. J Cell Biol 1993; 121: 439-448. 